\title{
IS SEX MAINTAINED TO FACILITATE OR MINIMISE MUTATIONAL ADVANCE?
}

\author{
J. T. MANNING \\ Department of Zoology, University of Liverpool, England
}

Received 31.x.75

\section{SUMmary}

There are two alternative hypotheses for the selective advantages of sex:

(i) The "Fisher-Muller" model : sex facilitates evolutionary adaptation to changing environments.

(ii) The "Ratchet" model: sex minimises the mutational load.

The relative importance of these hypotheses is discussed with reference to (a) comparative data on sexual and asexual reproduction, $(b)$ the timing of sex in species with asexual/sexual alternation, (c) the advantages of haploid/ diploid alternation, $(d)$ the disadvantage associated with the recombinational load.

It is concluded that the Ratchet model may well be the major mechanism which maintains sex.

\section{INTRODUGTION}

THE advantages of sexual reproduction, at both the population and individual level, have recently become a matter of controversy (reviewed by Maynard Smith, 1971; Felsenstein, 1974). Two major hypotheses for the selective advantage of sex have been advanced:

(1) The "Fisher-Muller" model (Fisher, 1930; Muller, 1932): this hypothesis suggests recombination results in increased genetic variance. As a result some offspring have higher numbers of favourable mutations than would be expected in a comparable asexual population. Sex, acting with selection, therefore accelerates evolutionary adaptation by facilitating mutational advance.

(2) The "Ratchet" model (Muller, 1964): this hypothesis agrees with the Fisher-Muller model in that it assumes recombination results in increased genetic variance. Muller points out, however, that an additional consequence of this increased variance is the production of some offspring with lower numbers of deleterious mutations than would be expected in a comparable asexual population. Sex, acting with selection, therefore reduces the mutational load by minimising mutational advance.

Theorists attempting to assign an immediate (one generation) advantage to sex (Maynard Smith, 1971; Williams and Mitton, 1973), have assumed the major advantage derives from the production of a greater fitness variance in the progeny. Williams and Mitton suggest that in each generation a few sexually produced offspring are " extraordinarily fit" in comparison with asexually produced progeny. Both the Fisher-Muller and Ratchet hypotheses are capable of increasing fitness variance. The former predicts the extraordinarily fit individuals combine a relatively large number of favour- 
able mutations. The latter suggests these individuals are relatively free of deleterious mutations.

It is of course possible that individuals may possess favourable mutations and a low mutation load. In this sense the models are not mutally exclusive. The purpose of this paper, however, is to discuss the relative importance of these two hypotheses in the maintenance of sex at the level of the individual.

The following points are argued: (1) the Ratchet model will be most important in species in which the environment is predictable, or is perceived as being predictable. The Fisher-Muller model will be most important in unpredictable environments. As comparative data indicate sex is universal in the former group of species and often lost or poorly represented in the latter, it follows the "Ratchet" model is the more important. (2) In those species with asexual/sexual alternation, the timing of sex may be explained by the interaction of the Ratchet model and density dependent factors. (3) The advantages derived from haploid/diploid alternation may well be similar to those associated with the Ratchet model. (4) The Ratchet model (but not the Fisher-Muller hypothesis) may explain why the generation of a recombinational load does not cause sex to be lost.

\section{ENVIRonmental PREDictability AND THE ogGurRENGE OF SEX}

In the long-term history of any group new evolutionary innovations must be important. In terms of immediate advantage, however, such innovations do not seem to be common enough to be of much significance in the maintenance of sex. When considering the relative importance of the Ratchet and Fisher-Muller hypotheses it must be first pointed out that most mutations are deleterious (Fisher, 1932). Any species which is well adapted to its environment will have a wild type which has a high level of fitness. When the environment is stable, this high level of fitness will also be stable. In such an environment an individual has the best chance of survival if it closely resembles its parent (Maynard Smith, 1971). The Ratchet model facilitates the removal of genotype changes and therefore will be most important in these species. Many groups have, however, evolved methods of making themselves independent of variations in the external environment. Such groups employ both behavioural and physiological buffering mechanisms. The former have reached their greatest development in taxa with large discrete nervous systems, well-developed sense organs and a high level of mobility. Physiological buffering extends to the control of $p \mathrm{H}$, osmotic pressure and body temperature. Levins (1968) has argued that these taxa experience their environment as uniform or "fine grained " in space and time. I suggest that in these species, mutations will have a very low probability of sustaining an advantage relative to the wild type. Homeostasis reaches its greatest development in the vertebrates. In particular, birds, mammals and reptiles have well-developed behavioural and physiological buffering mechanisms. Amphibians and fish are less well buffered against the environment. Homeostatic mechanisms are not so well developed in the invertebrates. However, the Cephalopoda are highly mobile and have a well-developed nervous and sensory system. The Insecta are also behaviourally buffered by their ability to fly, but this is reduced by their virtually immobile larval stage (Levins, 1968). Of the above taxa, the birds, mammals, reptiles and cephalopods are exclusively sexual, while sex is predominant in amphibians, 
fish and insects. This suggests the most important function of sex is to reduce the mutation load. Support for the above speculation comes from the observations of Selander and Kaufman (1973). These authors have pointed out that vertebrates show much less enzyme and protein variation than invertebrates. This could be a consequence of the operation of the Ratchet mechanism in the former group.

Behavioural and physiological buffering is poorly developed in the following groups: Bacteria, Protozoa, Porifera, Coelenterata, Platyhelminthes, Bryozoa and Annelida. These taxa perceive their environment as " coarse grained" in space and time (Levins, 1968). In the plants, behavioural buffering is limited, as it is in the Porifera and Coelenterata, by their fixed mode of life. However, increasing complexity and size may be expected to offset this somewhat through the sequence: Algae, Fungi, Bryophyta, Pteridophyta, Gymnospermae and Angiospermae. If the Fisher-Muller hypothesis is important in the maintenance of sex, the above groups should be predominantly sexual. Most of these taxa are however asexual or have marked asexual/sexual alternation. The Bacteria have parasexual processes but are predominantly asexual. Many Protozoan groups are apparently exclusively asexual. Of the remainder, most are isogamous and therefore sex does not incur a 50 per cent disadvantage relative to asexual reproduction (Manning, 1975). Despite this, asexual/sexual alternation is common in the Protozoa. Reproduction in the Porifera may be asexual or sexual, but the latter has not yet been demonstrated in many species. In the Coelenterata, Platyhelminthes, Bryozoa, Annelida and throughout the plant kingdom, asexual/sexual alternation is well developed.

This short review indicates the Ratchet model may well be more important than the Fisher-Muller hypothesis in the maintenance of sex.

\section{Asexual/sexual alternation}

Williams and Mitton (1973) have suggested that species showing asexual/sexual alternation should provide clues to the short-term advantages of sex. This alternation is found in the Protista, Coelenterata, Rotifera, Aphids and a number of parasitic groups. The timing of sex and the environmental conditions are important in any model which seeks to account for the maintenance of sex. Williams and Mitton in their "Aphid-Rotifer" model suggest this timing is essentially correlated with changes in the nature of the environment. Changes of this kind result from migrations of the individuals involved to new environments. This interpretation does not support an important role for the Ratchet mechanism in the maintenance of sex. I suggest, however, that the timing of sex in these species is correlated with a change in the intensity of selection and not its nature. That is, sex is associated with predictable decreases in population density. Models attempting to show an immediate advantage for sex have assumed it increases the fitness variance of progeny. Asexual reproduction produces more uniform progeny. The latter have an average fitness. Some sexually produced individuals are, however, extraordinarily fit. Sex will therefore be favoured by any factor which confines survival to a small proportion of individuals at the top of this fitness distribution. When the intensity of selection is constant from generation to generation, then population density will also remain constant. In these conditions Williams and Mitton (1973) 
have shown an increase in fecundity will confine survival to an even smaller fraction of individuals at the top of the fitness distribution (fig. 1). However, fluctuation in numbers, rather than constancy, is the rule (Huxley, 1974). This fluctuation may be irregular, or it may be cyclic and regular. When
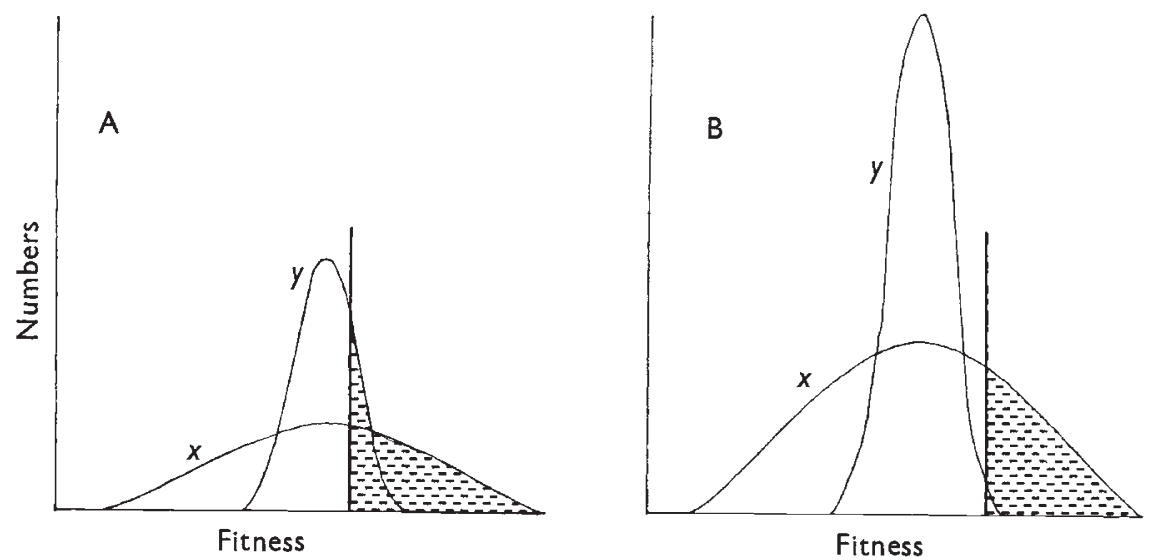

Frg. 1.-An increase in fecundity (IB over 1A) results in an increase in the proportion of survivors (dashed area) of sexually reproduced progeny (curve $\mathrm{X}$ ) relative to asexually reproduced progeny (curve $\mathrm{Y}$ ) in each generation.

selection intensity increases, numbers decline. In these circumstances selection ensures only the extraordinarily fit will survive. A decrease in population density will favour sexual reproduction, an increase will favour asexual reproduction (fig. 2).

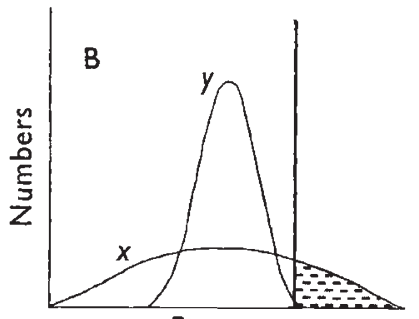

Fitness

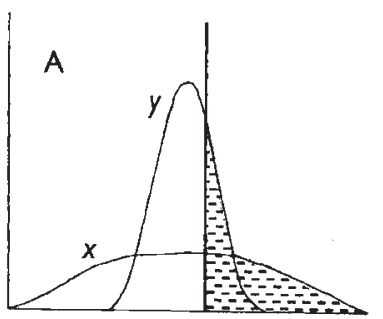

Fitness

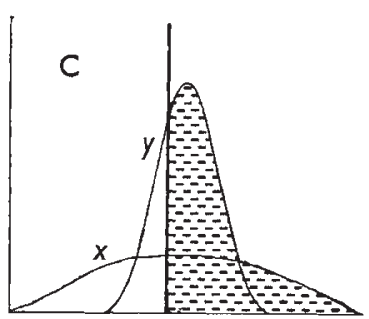

Fitness

Fig. 2.-A reduction in the number of survivors (dashed area) from one generation (2A) to the next (2B), results in an increased proportion of sexually reproduced progeny (curve $\mathrm{X}$ ) relative to asexually reproduced progeny (curve $\mathrm{Y}$ ). An increase in the number of survivors from one generation (2A) to the next $(2 \mathrm{C})$ results in a decrease in the proportion of sexually reproduced progeny $(\mathrm{X})$ relative to asexually reproduced progeny $(\mathrm{Y})$.

Virtually all species with asexual/sexual alternation are subject to marked changes in the intensity of selection. Sex commonly occurs immediately before a substantial reduction in population density. Micro-organisms suffer substantial reduction of their numbers during the winter, before which period sex occurs. In addition, sex can be stimulated to occur in many micro-organisms by the depletion of their culture medium. This depletion precedes a population crash. Many Rotifers inhabit temporary pools where 
populations are quickly built up. The spread to new pools represents a period of great egg loss. With sessile groups (e.g. Coelenterata) the spread to a new suitable substratum involves the loss of many progeny. Colonisation of the surrounding substratum is often by asexual means, but when greater distances are involved, then sex is common. A similar situation prevails in parasitic groups. Multiplication within the host is usually asexual. The spread to new hosts is often correlated with the timing of sex. The above model does not assume violent changes in the nature of selective pressures but rather in their intensity. It is therefore compatible with an important role for the Ratchet model in the maintenance of sex.

\section{HAPLOPhases AND SEXUAL REPRODUCTION}

Many plant and some animal species regularly produce haploid individuals. It has been known for some time that a mechanism essentially similar to the Ratchet model may be important in these species (White, 1954). The production of haplophases leads to the exposure of mutations without the protection of the diploid condition. In the plant kingdom the haploid stage or gametophyte is well developed in the Algae, Fungi, Bryophyta and Pteridophyta. Recombination precedes the production of the gametophyte. The Ratchet model predicts this will result in a proportion of gametophytes which combine few or no deleterious mutations. These extraordinarily fit individuals then go on to produce diploid sporophytes. These latter individuals may reproduce asexually before producing haploid spores by meiosis. Haploid/diploid alternation and the Ratchet model have very similar predicted results. That is, they both minimise mutational advance. In animal groups where haploidy occurs it is confined to the males. This phenomenon is particularly common in the Hymenoptera. Recent work has indicated that protein and enzyme variation is very low in this group (Metcalf, Marlin and Whitt, 1975).

\section{Recombination AND mutation loAd}

The existence of super genes is taken to be evidence that alternative environmental conditions are very often associated with one another (Felsenstein, 1974). Any break up of these linkage groups must reduce the mean fitness of the offspring. Recombination is therefore said to be associated with a "load". The existence of linkage in a group indicates that it does not exist in a completely unpredictable environment. When environments are predictable and constant then the recombinational load must be high. In such conditions the Fisher-Muller model does not provide a counterbalancing advantage which may exceed the disadvantage associated with this load. The Ratchet model, however, predicts this counterbalancing advantage could be the reduction in mutation load. This is the consequence of assuming sex adapts the individual to minimise genotype change and not to anticipate environmental change. Individuals will still be produced with major amounts of recombination in their co-adapted super genes, but these progeny are likely to be quickly eliminated by selection. The surviving sexually reproduced progeny are therefore likely to be more variable but carrying fewer mutations than a comparable asexual population. It is remarkable that such a possible mechanism was not apparently appreciated 
by Fisher. In fact, Fisher placed great emphasis on adaptations specifically designed to minimise fitness loss incurred by the "blizzard of predominantly unfavourable mutations" which all organisms sustain (Fisher, 1932). Such adaptations include the following: (1) The evolution of dominance, which results in an abundance of recessives amongst mutants. This is a common adaptation which maximises the fitness of the progeny on a one-generation basis. It has, however, the side effect of slowing the fixation of a mutation if that mutation becomes advantageous as a result of habitat change. Dominance therefore opposes, to some extent, long-term evolutionary change. (2) The reduction in fitness, seen as a result of inbreeding normally exogenous species possessing large numbers of recessives. (3) The existence of devices such as " the separation of the sexes" to ensure exogamy.

\section{Conclusion}

Two assumptions underlie much of the classical speculation on the advantages of sex. These are: (1) It is essentially concerned with evolution and (2) it produces many new genotype combinations and therefore must sustain its optimum advantage when conditions regularly change. These assumptions have much in common with each other and their virtually unchallenged popularity for many years is not surprising. Thus Dobzhansky (1972) has pointed out that the mechanism of natural selection was proposed by Blyth well before the Darwin-Wallace papers of 1858. However, the original papers referred to stabilising rather than directional selection, i.e. constancy rather than change. Selection appears therefore to be intuitively associated with evolutionary change, despite the modern awareness of its importance in the stabilisation of gene frequencies. In addition, the advantage of producing new genotype combinations in violently fluctuating conditions seems, at first sight, to agree well with the type of habitat occupied by organisms showing asexual/sexual alternation. These assumptions underlie many excellent works on the advantages of sex. The first suggestion, however, concerns itself with that apparent rare event, the favourable mutation. The second with the "scrambling" of successful genotypes to produce new equally successful gene combinations. The existence of linkage and predominantly unfavourable mutations suggest it is more likely that sex is concerned with a struggle by organisms to maintain the integrity of their informational molecules.

Acknowledgments.-I am very grateful to the anonymous referee of this paper for a number of constructive criticisms.

\section{REFERENGES}

Dobzhansky, т. 1972. Natural selection and mankind, in The Structure of Human Populations, pp. 213-233. Oxford University Press.

FELSENSTEIN, J. 1974. The evolutionary advantage of recombination. Genetics, 78, 737-756. FISHER, R. A. 1930. The Genetical Theory of Natural Selection. Clarendon Press, Oxford.

FISHER, R. A. 1932. The bearing of genetics on theories of evolution. Sci. Prog., Oxf., 27, 273-287.

HUXLEx, J. 1974. Evolution-The Modern Synthesis. Allen \& Unwin, London. Levins, R. 1968. Evolution in Changing Environments. Princeton University Press, Princeton, New Jersey. 
MANNING, J. T. 1976. Anisogamy and the cost of sexual reproduction: are they separate phenomena? F. Theor. Biol., 55, 393-395.

MAYNARD SMrth, J. 1971. What use is sex? f. Theor. Biol., 30, 319-335.

METCALF, R. A., MARLIN, J. C., AND WHITT, G. s. 1975. Low levels of genetic heterozygosity in Hymenoptera. Nature, 257, 792-794.

MULLER, H. J. 1932. Some genetic aspects of sex. Am. Nat., 66, 118-138.

MULLER, H. J. 1964. The relation of recombination to mutational advance. Mutation Res., $1,2-9$.

SELANDER, R. K., AND KAURMAN, D. W. 1973. Genic variability and strategies of adaptation in animals. Proc. Nat. Acad. Sci., U.S.A., 70, 1875-1877.

whrTe, M. J. D. 1954. Animal Cytology and Evolution. Cambridge University Press, Cambridge.

williams, G. C., AND mitron, J. B. 1973. Why reproduce sexually? 7. Theor. Biol., 39, $545-554$ 